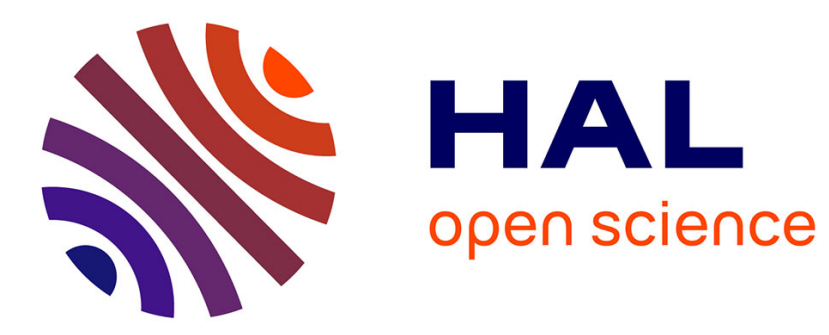

\title{
Multi-scale modeling of the viscoelastic behavior of 3D woven composites
}

\author{
Martin Hirsekorn, Lionel Marcin, Thierry Godon
}

\section{To cite this version:}

Martin Hirsekorn, Lionel Marcin, Thierry Godon. Multi-scale modeling of the viscoelastic behavior of 3D woven composites. Composites Part A: Applied Science and Manufacturing, 2018, 112, pp.539-548. 10.1016/j.compositesa.2018.07.011 . hal-01858178

\section{HAL Id: hal-01858178 https://hal.science/hal-01858178}

Submitted on 20 Aug 2018

HAL is a multi-disciplinary open access archive for the deposit and dissemination of scientific research documents, whether they are published or not. The documents may come from teaching and research institutions in France or abroad, or from public or private research centers.
L'archive ouverte pluridisciplinaire HAL, est destinée au dépôt et à la diffusion de documents scientifiques de niveau recherche, publiés ou non, émanant des établissements d'enseignement et de recherche français ou étrangers, des laboratoires publics ou privés. 


\title{
Multi-scale modeling of the viscoelastic behavior of 3D woven composites
}

\author{
Martin Hirsekorn $^{\mathrm{a}}$, Lionel Marcin ${ }^{\mathrm{b}}$, Thierry Godon ${ }^{\mathrm{c}}$ \\ ${ }^{a}$ Onera - The French Aerospace Lab, 29 avenue de la Division Leclerc, BP 72, 92322 Châtillon Cedex, France \\ ${ }^{b}$ Safran Tech, rue des Jeunes Bois, Châteaufort, CS 80112, 78772 Magny les Hameaux, France \\ ${ }^{c}$ Safran Aircraft Engines, Rond point René Ravaud, Fr7550 Moissy Cramayel, France
}

\begin{abstract}
A method is presented to predict numerically the homogenized viscoelastic behavior of $3 \mathrm{D}$ woven composites using only its constituents' behavior. It is based on elastic homogenizations applied to the Laplace-Carson transform of the time-dependent viscoelastic behavior of the constituents. Two scale chages are necessary: from micro- to meso-scale to obtain the homogenized behavior of the consolidated yarns, and from mesoto macro-scale. The temperature and cure dependent viscoelastic behavior of the matrix is identified from experimental data, using the time-temperature superposition principle with the cure dependent glass transition temperature as reference temperature. The meso-scale representative unit cell of the composite is extracted from X-ray microtomography images. The homogenized viscoelastic behavior is used to calculate the evolution of the apparent moduli of the composite with temperature between $-50^{\circ} \mathrm{C}$ and $200^{\circ} \mathrm{C}$. The results are in good agreement with experimental data over the temperature range where the matrix behavior was properly identified.
\end{abstract}

Key words: A. 3-dimensional reinforcement, B. Thermomechanical, B. Stress relaxation, C. Computational modeling

\section{Introduction}

During the manufacturing process of composites with thermosetting polymer matrices, residual stresses in the material cannot be avoided, because the thermosetting polymers are usually cured at much higher temperatures than the typical in-service temperature of the composite parts. These residual stresses may cause damage and influence its evolution $[1,2,3,4]$ and change the final shape of composite parts $[5,6,7,8,9]$. The major part of the residual stresses is due to a mismatch in the thermal expansion coefficients of the constituent materials (fibers and matrix), and thus generated during the cooling of the composite after the end of the curing cycle [5, 10,6]. Furthermore, a thermosetting polymer shrinks during curing [11, 12], which may also contribute to the formation of residual stresses $[13,10,6]$.

\footnotetext{
*Corresponding Author. Email: martin.hirsekorn@onera.fr 
While the mechanical behavior of carbon or glass fibers is linear elastic in a good approximation and almost independent of temperature, at least below the glass transition temperature $\left(T_{g}\right)$ of typical thermosetting polymers (usually not exceeding about $200^{\circ} \mathrm{C}$ ), the mechanical behavior of the polymer matrix depends strongly on temperature and on the degree of cure $[14,15,16,17,18,12]$. Moreover, it is time dependent, i.e., creep and relaxation phenomena are observed, which may have a significant impact on the residual stresses and the final shape of composite parts $[13,19,20,21]$. As a consequence, residual stresses can be reduced by adapting the cure cycle such that relaxation phenomena are intensified [13, 22].

Since in the aeronautical industry highly precise part shapes are required, it is currently common practice to adapt the mold shape several times until the composite part fits the shape tolerances. This procedure of designing a composite part is very expensive, but can be greatly optimized if precise modeling tools are available that predict the generation of residual stresses and the final shape of composites with complex reinforcements, such as three-dimensional (3D) interlock weavings. To improve the accuracy of currently available modeling tools, it is necessary to take into account creep and relaxation and their dependence on temperature and degree of cure during the modeling of the curing and cooling process. The experimental characterization of a composite material required to determine the model parameters is complex and expensive, as 3D strain rate, temperature, and cure dependent mechanical behaviors have to be measured. Furthermore, these properties have to be re-identified, each time the reinforcement architecture is changed. Significant cost reduction is possible if only the constituent materials are fully characterized and the composite properties are predicted using multi-scale models.

At small strains, creep, relaxation, and strain rate dependence of polymer resins are well described by viscoelastic models of the generalized Maxwell type $[23,14,15,16]$. This kind of model was used by several authors to simulate the generation of residual stresses in composites with both thermosetting and thermoplastic polymer matrices [24, 25, 26, 20, 27]. In all these works, the time-temperature superposition principle used, as it was shown to describe well the temperature dependence of the viscoelastic behavior of polymers $[14,15,16,28]$. The influence of the degree of cure was introduced in some works as an explicit dependence of the relaxation times on degree of cure $[14,16,27]$, but it can also be modeled through a cure dependent $T_{g}$ used as reference temperature in the time-temperature superposition principle [15, 25, 28, 29].

In the present study we focus on composites with $3 \mathrm{D}$ woven reinforcements $[30,31,32]$. This type of composites became an interesting option for industrial applications since the development of modern looms capable of weaving complex reinforcement structures [30, 31]. It is therefore possible to manufacture complex composite parts with very few assembling steps and thus short production times [33], reducing the number of joints, which are often critical for damage onset. Moreover, composites with 3D woven reinforcements are less prone to delamination and thus more resistant to damage caused by impact [30, 34, 35] compared to multi-layer composites of $2 \mathrm{D}$ weavings or unidirectional (UD) plies. In composites with woven reinforcements, usually three characteristic scales are distinguished [36]: the micro-scale, at which each fiber 
and the matrix is modeled individually, the meso-scale, at which the reinforcement architecture is modeled and yarns consisting of several thousand fibers are treated as homogeneous material, and the macro-scale, at which the structure is modeled and the composite material is treated as homogeneous.

Modeling the generation of residual stresses in these materials is therefore a multi-scale problem, and suitable techniques are needed to determine the homogenized behavior of a heterogeneous material at the next higher scale. A common technique to calculate the homogenized behavior of heterogeneous viscoelastic materials is to apply the Laplace-Carson (LC) transform to the integral [37, 38, 39, 40, 41] or the differential $[42,43]$ formulation of the viscoelastic behavior to obtain a linear relationship between the transformed stress and strain history (more details will be given in section 3). In this way, classical elastic homogenization methods can be used to determine the homogenized viscoelastic behavior.

In the present article, a homogenization strategy for composites with 3D woven reinforcements is presented based on the LC transform. It allows obtaining the viscoelastic behavior of the composite material from the behavior of its constituents, including the dependence on temperature and degree of cure. In section 2, the formulation of the viscoelastic models used in this work is presented. A 3D generalization of the viscoelastic model developed by Courtois et al. [29] for the polymer matrix of the studied composite is given in section 2.2. The proposed homogenization strategy is described in section 3 and validated numerically at the microscopic scale by comparison to full-field Finite Element (FE) calculations. In section 4, the strategy is applied to the case of a 3D woven composite using a representative unit cell (RUC) of the reinforcement architecture obtained from X-ray micro-computed tomography ( $\mu$-CT). The model is again validated numerically by comparison to full-field FE calculations. The obtained homogenized viscoelastic behavior of the composite is used to calculate the temperature dependence of the apparent elastic moduli of the composite. The results are compared to experimental observations.

\section{Viscoelastic constitutive model}

\subsection{General anisotropic formulation}

To model the viscoelastic behavior of the composite and its constituents at the different scales (yarns, matrix) the generalized Maxwell model is used. This model can be related to the orthotropic generalization [44] of Schapery's [45] integral form of viscoelastic behaviors: For a material that is in a completely relaxed state at the time $t_{0}$, the stress response to a strain history starting at $t_{0}$ can be written in the integral form

$$
\underline{\sigma}(t)=\int_{t_{0}}^{t} \underline{\underline{E}}\left(t-t^{\prime}\right): \underline{\dot{\varepsilon}}\left(t^{\prime}\right) d t^{\prime}
$$

where $\underline{\underline{E}}$ is the relaxation stiffness tensor (a 3D generalization of the relaxation modulus) and $\underline{\dot{\varepsilon}}\left(t^{\prime}\right)$ is the strain rate at a time between $t_{0}$ and $t$. In Eq. (1) and in the following, single underlining indicates second order tensors, double underlining indicates fourth order tensors, and the colon indicates contraction over two indices. 
The relaxation modulus is often written in terms of a Prony series $[14,15,16]$, which can be done in the same way for $\underline{\underline{E}}$ :

$$
\underline{\underline{E}}(t)=\underline{\underline{E}}_{\infty}+\sum_{k=1}^{N} \underline{\underline{E}}_{k} \mathrm{e}^{-\frac{t-t_{0}}{\tau_{k}}}
$$

where $\tau_{k}$ is called the relaxation time of the $k^{\text {th }}$ relaxation mechanism. Using this expression, the integral form resolves to

$$
\underline{\sigma}(t)=\underline{\underline{E}}_{0}: \underline{\varepsilon}(t)-\sum_{k=1}^{N} \underline{\underline{E}}_{k}: \varepsilon_{k}^{v e}(t)
$$

with the instantaneous elastic stiffness tensor

$$
\underline{\underline{E}}_{0}=\underline{\underline{E}}_{\infty}+\sum_{k=1}^{N} \underline{\underline{E}}_{k}
$$

The tensorial internal variables $\underline{\varepsilon}_{i}^{v e}$ associated to the relaxation times $\tau_{i}$ are called history variables [44]. Their use avoids the integration over the strain history (Eq. 1).

The evolution in time of the $\underline{\varepsilon}_{i}^{v e}$ is given by differential equations of the form

$$
\underline{\dot{\varepsilon}}_{k}^{v e}(t)=\frac{1}{\tau_{k}}\left(\underline{\varepsilon}(t)-\underline{\varepsilon}_{k}^{v e}(t)\right)
$$

They describe the evolution in time of the strains of the viscoelastic elements in a generalized Maxwell model. This means that under the assumption of a Prony series description for the relaxation stiffness, the integral form is equivalent to a generalized Maxwell model, with each term in the Prony series corresponding to one viscoelastic Maxwell element (with a 3D elastic behavior described by the tensor $\underline{\underline{E}}_{k}$ ). This form of an anisotropic generalized Maxwell model is used in the following to model the viscoelastic behavior of the composite and its constituents. The composite behavior is orthotropic, the yarns obey transverse isotropy around the fiber axis, and the matrix is isotropic. In the respective viscoelastic models, the tensors $\underline{\underline{E}}_{k}$ and $\underline{\underline{E}}_{\infty}$ take the appropriate form with 9 (orthotropy), 5 (transverse isotropy), or 2 (isotropy) independent coefficients.

\subsection{Specification for the polymer matrix}

The polymer used as a matrix in the composite material described in section 4 is an isotropic material. The fourth order tensors in Eq. (3) can thus be written as

$$
\begin{aligned}
& \underline{\underline{E}}_{k}=K_{k}\left(\frac{1}{3} \underline{\mathbf{1}} \otimes \underline{\mathbf{1}}\right)+G_{k}\left(\underline{\underline{\mathbf{1}}}-\frac{1}{3} \underline{\mathbf{1}} \otimes \underline{\mathbf{1}}\right) \\
& \underline{\underline{E}}_{\infty}=K_{\infty}\left(\frac{1}{3} \underline{\mathbf{1}} \otimes \underline{\mathbf{1}}\right)+G_{\infty}\left(\underline{\underline{\mathbf{1}}}-\frac{1}{3} \underline{\mathbf{1}} \otimes \underline{\mathbf{1}}\right)
\end{aligned}
$$

where $\underline{\mathbf{1}}$ is the second order identity tensor and $\underline{\underline{\mathbf{1}}}$ the fourth order identity tensor with minor symmetry.

Courtois et al. [29] carried out relaxation tests on fully and partially cured specimens of the epoxy resin used in the studied composite at different temperatures below its glass transition temperature $\left(T_{g}\right)$. Based 
upon their data, they proposed a 1D model for the tensile viscoelastic behavior of the resin as a function of temperature and degree of cure. In a composite material, the matrix is exposed to complex 3D strain states. To predict the homogenized behavior of the composite, we therefore need a full 3D model of the viscoelastic behavior of the matrix. If we use 1D model of Courtois et al. [29] directly to model the evolution of the tensile modulus in a 3D model, this would imply the assumption of a constant Poisson ratio during relaxation. However, it was observed by Tschoegl et al. [46], O'Brien et al. [47] that the Poisson ratio increases during relaxation approaching values close to 0.5 for a fully relaxed material at high temperatures. We therefore assume that the viscoelastic strain is isovolumetric, which corresponds to setting all $K_{k}$ in Eq. (6) to zero (and, as a consequence from Eq. (4), $K_{0}=K_{\infty}$ ).

Under this assumption, the stress relaxation at a constant tensile strain (with all other strain components unconstrained) is accompanied by an increasing transverse strain, thus reproducing the phenomena observed in $[46,47]$. This implies that, while the 1D model was identified under the assumption of a constant strain, this is no longer the case for the 3D strain tensor. We therefore re-identified the model parameters by simulating the experiments of Courtois et al. [29] using the 3D model with the assumption of isovolumetric viscoelastic strain. This means that instead of identifying the tensile moduli $E_{k}$ of the Maxwell elements, we identify their shear moduli $G_{k}$.

Courtois et al. [29] proposed a continuous function to approximate the tensile viscous moduli as a function of the relaxation times $\tau_{k}$. In the present work, we use the same continuous function to describe the viscous shear moduli $G_{k}$ as a function of the relaxation times $\tau_{k}$ :

$$
G\left(\tau_{k}\right)=A \cdot \exp \left(-\left(\frac{\log _{10} \tau_{k}-\log _{10} \tau_{\text {peak }}}{l_{\text {peak }}}\right)\right)+\frac{B}{2} \cdot\left(1-\operatorname{erf}\left(\frac{\log _{10} \tau_{k}-\log _{10} \tau_{\text {peak }}}{l_{\text {peak }}}\right)\right)
$$

where $\tau_{\text {peak }}, l_{\text {peak }}$, and $A$ are the position, width, and height of a Gauss-curve like peak on the logarithmic relaxation time scale, and $B$ is the height of the plateau of the function towards short relaxation times. erf is the Gaussian error function. The relaxation times $\tau_{k}$ are given in minutes.

The temperature dependence of the viscoelastic behavior is modeled by means of the principle of timetemperature superposition. An Arrhenius model is used for the shift factors [15, 48, 29]:

$$
a_{T}(T, c)=\frac{H(c)}{R \cdot \ln 10}\left(\frac{1}{T}-\frac{1}{T_{g}(c)}\right)
$$

with the cure dependent $T_{g}$ as reference temperature. $H$ is an activation energy, which was found to decrease linearly with the degree of cure [29], and $R$ the idealized gas constant.

The cure dependence is introduced via $T_{g}$ by means of the DiBenedetto equation [49]

$$
\frac{T_{g}(c)-T_{g 0}}{T_{g 1}-T_{g 0}}=\frac{\lambda c}{1-c(1-\lambda)}
$$

where $T_{g 1}$ is the glass transition temperature of the fully cured resin, and $T_{g 0}$ is the glass transition temperature of the uncured resin. $c$ designates the degree of cure. 
The cure and temperature dependent relaxation times are thus given by:

$$
\log _{10} \tau(T, c)=\log _{10} \tau\left(T_{g}(c)\right)+a_{T}\left(T, T_{g}(c)\right)
$$

In this model, the instantaneous elastic modulus is independent of the degree of cure, as confirmed experimentally by $[14,16]$. However, since the relaxation curves are shifted towards longer relaxation times for increasing degrees of cure, the apparent elastic modulus (slope of the stress-strain curve over a given strain range in the linear domain) increases with cure, and the Poisson ratio decreases from values close to 0.5 at the gel point to the values observed for the fully cured resin at room temperature, as observed by $[47]$.

The parameters of the model are summarized in Table 1. Note that they are different from those obtained by Courtois et al. [29], because in the present work, we introduce viscoelasticity into the shear behavior (instead of the tensile behavior) to model the same experimental data, which implies that the 3D strain tensor is not constant during the relaxation phases. The model is numerically implemented with one Maxwell element per decade of relaxation times [29], which is sufficient to well reproduce the observed relaxation curves. The relaxation times are chosen such that they reach from the highest tested strain rate at the coldest temperature (shortest shifted relaxation time) to the longest tested relaxation time at the highest temperature (longest shifted relaxation time).

\section{Viscoelastic homogenization}

\subsection{Material behavior in the Laplace-Carson space}

The Laplace-Carson (LC) transform of a function $f$ in time $t$ is defined as

$$
\hat{f}(p)=p \int_{0}^{\infty} f(t) \mathrm{e}^{-p t} d t
$$

Applied to the integral form of a viscoelastic behavior (Eq. 1), a linear relation between the transformed stress and strain is obtained:

$$
\underline{\hat{\sigma}}(p)=\underline{\underline{\hat{E}}}(p): \underline{\hat{\varepsilon}}(p)
$$

This means that in the transformed space, the viscoelastic constitutive equation corresponds to Hooke's law with stiffness tensors depending on the transformation parameter $p$. Therefore, common homogenization techniques for elastic materials can be used in the transformed space to calculate the LC transform of the homogenized viscoelastic behavior. To this result, the inverse LC transform has to be applied in order to determine the homogenized viscoelastic behavior in the time space.

The LC transform of a relaxation stiffness written in terms of a Prony series (Eq. 2) is given by

$$
\underline{\underline{\underline{E}}}(p)=\underline{\underline{E}}_{\infty}+\sum_{k=1}^{N} \frac{p}{p+\frac{1}{\tau_{k}}} \underline{\underline{E}}_{k}
$$


For the special values of $p=0$ and $p=\infty$, the relaxed $\left(\underline{\underline{\hat{E}}}(0)=\underline{\underline{E}}_{\infty}\right)$ and the instantaneous stiffness $\left(\underline{\underline{E}}(\infty)=\underline{\underline{E}}_{0}\right.$, see Eq. 4) are obtained. This means that the relaxed and the instantaneous stiffness tensor of the homogenized material can be calculated directly by elastic homogenization using the relaxed and instantaneous stiffness tensors of the constituents, respectively. The LC transform is only needed to calculate the $\underline{\underline{E}}_{k}$ of the viscoelastic Maxwell elements.

\subsection{Homogenization strategy}

The relaxation spectrum of the matrix can well be described by the continuous function given in Eq. (7). The function is sufficiently smooth that one relaxation time per decade is enough to well reproduce the experimentally observed relaxation curves [29]. We suppose in the following that the yarns and the composite also have a continuous relaxation spectrum that can be described by one relaxation time per decade. In this case, the model described in section 2 can be used in its anisotropic form to model the yarns and the composite, and we choose the same relaxation times for the yarns and the composite as for the matrix. Consequently, the homogenized model will cover the same range of temperatures and strain rates as the matrix model. This approach is similar to those presented in [37, 39].

Lévesque et al. [38] discussed the accuracy of the numerical inversion of the LC transform and proposed an algorithm that determines the relaxation times of the homogenized material such that the accuracy of the inverse LC transform meets an acceptance criterion. According to Lévesque et al. [38], a priori definition of the relaxation times affects the quality of the inversion of the LC transform. However, if the relaxation spectrum for both the constituents and the composite is continuous and sufficiently smooth, the choice of the same, regularly spaced, relaxation times will provide an accurate reproduction of the homogenized relaxation curves, if the stiffness tensors of the Maxwell elements are appropriately determined. This is not obvious, since the underlying morphology of the multi-phase material may strongly modify the resultant spectrum shape [42].

We therefore control for the conditioning of the inverse LC transform and propose an optimum choice of the parameters $p$, at which the transformed stiffnesses should be calculated if the same regularly spaced relaxation times are used for the composite and all its constituents (section 3.3). To validate the hypothesis of continuous homogenized spectra and the accuracy of the proposed homogenization method, the homogenized viscoelastic behavior is compared with $\mathrm{FE}$ simulations of the viscoelastic behavior of the full heterogeneous RUC used for homogenization (section 3.4 for the micro-scale and section 4.2 for the meso-scale).

The proposed homogenization strategy is thus summarized as follows:

- Calculate the LC-transformed stiffness tensors for each constituent for $M$ different values of $p$ using Eq. (13)

- Calculate the homogenized LC-transformed stiffness tensors for each $p$ by elastic homogenization 
- Calculate the homogenized relaxed stiffness tensor $\underline{\underline{E}}_{\infty}$ by elastic homogenization

- Calculate the homogenized $\underline{\underline{E}}_{k}$ by solving the equation system given by Eq. (13) using the homogenized $\underline{\underline{E}}(p)$ and $\underline{\underline{E}} \infty$

The third point of the strategy is actually equivalent to the second point for $p=0$, and the elastic homogenization of the instantaneous stiffness is equivalent to the second point for $p=\infty$. The last step corresponds to the numerical inversion of the LC transform. Its accuracy thus depends on the conditioning of the equation system Eq. (13). In order to calculate the $N$ tensors $\underline{\underline{E}}_{k}$, this equation system needs full rank. Therefore, the $\hat{E}(p)$ of each constituent have to be calculated for at least $N$ different values of $p$, i.e., $M \geq N$. If more $p$ are used, the Moore-Penrose pseudoinverse of the transformation matrix is calculated to determine the homogenized $\underline{\underline{E}}_{k}$.

\subsection{Conditioning of the inverse Laplace-Carson transform}

The conditioning of the inverse LC transform is analyzed for a homogenization starting from a micro-scale representative unit cell (RUC) of a yarn. At this scale, the RUC is relatively simple and thus computational costs are low. A hexagonal periodic distribution of parallel fibers was chosen as RUC for the yarns (Figure 1), because it yields a transverse isotropic elastic behavior. The fiber volume fraction in the RUC was fixed such that the overall fiber volume fraction and the warp to weft yarn weight ratio of the composite specimens studied in section 4.3 is obtained with the mesoscopic composite model used in section 4.1. In the following, the method is demonstrated for the case of a warp yarn (74.6\% fiber volume fraction).

A FE mesh of the hexagonal unit cell is generated using second order prismatic elements (15 nodes) with reduced integration. The unit cell for a warp yarn shown in Figure 1 contains 3068 elements, 656 in the fiber and 2412 in the matrix. The matrix discretization was chosen such as to ensure at least 6 matrix elements between two neighboring fibers. Since the fiber is much stiffer than the matrix, the strain field in the fiber is relatively homogeneous. The element size in the fiber can thus be chosen much larger than in the matrix. Periodic boundary conditions (PBC) are applied in the three directions of periodicity in the plane perpendicular to the fiber, and in fiber direction. The FE problems are solved using an implicit scheme solved using the Newton-Raphson method. Convergence is reached if the norm of the internal forces is smaller than $10^{-5}$ times the norm of the external forces.

For each elastic homogenization, the RUC is loaded such that its average strain corresponds successively to the 6 elementary strains (only one component $\varepsilon_{k l}=\varepsilon_{l k}$ of the strain tensor is non-zero). The components of the homogenized elastic stiffness tensor $\underline{\underline{E}}$ are then obtained from average stress response $\sigma_{i j}$ to the average strain $\varepsilon_{k l}$ as $E_{i j k l}=\sigma_{i j} / \varepsilon_{k l}$. This method is used to calculate the homogenized transformed stiffness tensors $\underline{\underline{E}}\left(p_{i}\right)$ for different choices of $p_{i}, i \in\{1, \ldots, M\}$ with $M \geq N$. For each $p_{i}$, the transformed matrix stiffness is obtained from Eq. 13. Since the fiber behavior is elastic, its transformed stiffness is independent of $p$ 
and equal to its stiffness in time space. The elastic properties of the fiber (transverse isotropic) are given in Table 2 .

From the homogenized $\hat{E}\left(p_{i}\right)$, the parameters of the homogenized generalized Maxwell model are obtained by solving the equation system (13). A measure for the precision of the solution is the condition number of the matrix $\mathcal{L}_{i k}=\frac{p_{i}}{p_{i}+\frac{1}{\tau_{k}}}$. It only depends on the relaxation times $\tau_{k}$ and the choice of the $p_{i}$. Therefore, the results presented in the following for the case of a micro-scale homogenization also apply to the mesoscopic scale (section 4).

A convienient choice for one $p_{i}$ is $p=\infty$, because $\underline{\underline{\underline{E}}}(\infty)$ corresponds to the instantaneous elastic stiffness (see Eq. 13). The homogenized $\underline{\underline{E}}(\infty)$ can therefore be obtained by simple elastic homogenization using the instantaneous stiffnesses of the constituents (see sections 3.1 and 3.2). This $p=\infty$ will be used in all of the following homogenizations. The other $p_{i}$ are chosen proportional to the inverse of the relaxation times, which depend on temperature $T$ and degree of cure $c$ through the shift factors $a_{T}$ (see Eq. 10). In this way, the $p_{i}$ depend on $T$ and $c$ through the negative shift factors $-a_{T}$. As a consequence, the matrix $\mathcal{L}$ becomes independent of $T$ and $c$. Since the stiffness tensors $\underline{\underline{E}}_{k}$ and $\underline{\underline{E}}_{\infty}$ of the polymer resin are also independent of $T$ and $c$ (and the fiber behavior is independent of $T$ and $c$ as well), the homogenized $\underline{\underline{E}}_{k}$ and $\underline{\underline{E}}_{\infty}$ are also independent of $T$ and $c$. The proposed homogenization technique (in which the same relaxation times are used for the homogenized material and for its constituents) thus transfers the $T$ and $c$ dependence of the constituents directly to the homogenized behavior, with same shift factors for the relaxation times.

It is therefore sufficient to carry out the homogenization at the reference temperature $T_{g}(c)$. One relaxation time per decade is chosen such that the whole strain rate and temperature range of the experiments presented in section 4.3 is covered $\left(\tau_{k}=10^{k} \mathrm{~min}, k \in\{-22, \ldots, 2\}\right.$ at $\left.T_{g}(c)\right)$. The simplest option ensuring that $\mathcal{L}$ is independent of $T$ and $c$, is to choose $p_{i}=\frac{1}{\tau_{i}}$ in addition to $p=\infty$. However, the resulting homogenized spectrum exhibits some oscillations in the shear moduli (Figure 2), which for the case of the transverse shear modulus even includes some negative values. Adding one larger and one smaller $p_{i}\left(10^{23}\right.$ and $\left.10^{-3}\right)$ has no notable influence on the results. If some information at intermediate times is added to the system by using $p_{i}=10^{i} \min ^{-1}, i \in\{\infty, 22.5,22.0,21.5, \ldots,-2.5\}$, the spectra become smooth and the condition number of the matrix $\mathcal{L}$ is reduced by more than a factor of 4 . The conditioning becomes even better, if the $p_{i}$ are limited to times that lie in between the relaxation times $\left(p_{i}=10^{i} \min ^{-1}, i \in\{\infty, 22.5,21.5,20.5, \ldots,-2.5\}\right)$. This choice of the $p_{i}$ ensures smooth spectra, and only a total of $M=N+3$ elastic homogenizations are necessary to obtain the homogenized viscoelastic behavior.

\subsection{Comparison between the homogenized behavior and FE simulations}

The homogenized behavior obtained with this choice of $p_{i}$ is validated by comparison with FE simulations using the heterogeneous RUC shown in Figure 1 with the polymer behavior presented in section 2.2 for the matrix and the properties given in Table 2 for the fiber. The temperature is set to $T_{g}(c)$ (which yields 
$a_{T}=0$ from Eq. 8). Accordingly, the relaxation times are set to $\tau_{k}=10^{k} \min , k \in\{-22, \ldots, 2\}$ at $T_{g}(c)$. An average strain of $0.1 \%$ is applied to the RUC within $10^{-17} \mathrm{~min}$ and held constant for $10^{4} \mathrm{~min}$ (in order to cover 21 orders of magnitude in time, a default range in the used FE software). The other average strain components are free. The average stress response of the RUC corresponds to the relaxation master curve over the simulated time scale. Tracing the free strain components in time allows to validate the evolution of the Poisson ratio during relaxation. The same FE mesh, $\mathrm{PBC}$, and convergence criteria are used as in the homogenization procedure (section 3.3).

Figure 3 shows that the homogenized behavior is in very good agreement with the full viscoelastic FE simulation for all load cases. We also observe that due to the isovolumetric viscous strain of the matrix, the Poisson effect increases during relaxation. The agreement over the whole relaxation time range confirms that the assumption of a continuous viscoelastic spectrum of the homogenized yarn material (section 3.2) was correct.

\section{Viscoelastic behavior of the composite}

\subsection{Representative Unit Cell}

In this section, the homogenization method presented in section 3 is used to calculate the viscoelastic behavior of a composite material with a 3D woven interlock reinforcement. Building a FE mesh whose element faces are consistent with the internal material geometry (interfaces between yarns in contact and between yarns and matrix pockets) is a difficult task [50], in particular for highly complex 3D reinforcements. On the other hand, voxelized meshes are appropriate to calculate homogenized elastic properties [51, 52] if the voxel size is chosen sufficiently small such that the volume fractions of warp and weft yarns and the matrix pockets are accurately represented. Since the proposed homogenization method is based on elastic FE calculations only, we use voxel meshes in the following to avoid the highly complex meshing task.

Voxel meshes can be built directly from $\mu$-CT images of the fibrous reinforcement in the composite $[53,54,55]$. By analyzing the gray scale levels of the $\mu$-CT images and the orientations of the grayscale gradients, each voxel can be assigned to either a matrix pocket or a specific warp or weft yarn. The resulting voxel mesh of a meso-scale RUC of the studied composite is shown in Figure 4. It contains a total of 373932 voxels (141 in warp direction, 102 in weft direction, and 26 in out-of-plane direction). The effective volume of the voxels assigned to the warp yarns, the weft yarns, and the matrix is calculated. The fiber volume fractions in the warp and weft yarns that are used in the following calculations are adjusted such that the overall fiber volume fraction is $59 \%$ and the weight ratio of the fibers in the warp and weft yarns is $79 / 21$. With the voxel mesh shown in Figure 4, the fiber volume fraction in the warp yarns is $74.6 \%$ and in the weft yarns $79.6 \%$. For each slice of the voxel mesh, the center of the cross-section of each yarn is determined. These points are used to build the yarn center line by spline interpolation. The fiber orientation in each 
yarn slice is then set to the direction of the tangent to the yarn center line in the respective slice. The elastic properties assigned to the yarn voxel elements are obtained by rotating the stiffness tensor assigned to the yarn such that the axial behavior $(\mathrm{L})$ is obtained in the local fiber direction.

The same homogenization method as presented in section 3.1 is used with the optimum choice of $p_{i}$ given in section 3.3. Each voxel is simulated by a linear hexahedral element ( 8 nodes) with full integration. The same convergence criterion for the implicit scheme is used as at the microscale. 3D periodic boundary conditions (PBC) are used at the meso-scale as well, even if the reinforcement geometry obtained from micro-CT is not perfectly periodic. However, mixed uniform boundary conditions (MUBC) as used by Hello et al. [54] on similar materials lead to an overestimation of the homogenized stiffness, because constant normal displacements are imposed on the RUC boundaries for the tensile load cases, which prohibit relative displacements of the yarns and matrix pockets in these directions. With PBC, we expect a slight underestimation of the composite stiffness, because the opposite ends of yarns with not perfectly periodic paths on the RUC boundaries are not attached entirely to each other. Since the voxel mesh is periodic due to its regular structure, the application of 3D periodic boundaries is straightforward.

\subsection{Numerical validation of the homogenized behavior}

The obtained homogenized viscoelastic behavior is compared to full FE simulations of the viscoelastic behavior of the RUC in the same way as at the microscopic scale (section 3.4), using the same mesh, boundary conditions, and convergence criteria as for the homogenization procedure (section 4.1). The results are shown in Figure 5. Very good agreement is observed for the tensile and shear relaxation curves in all directions, and for the evolution of the Poisson ratios in time, which shows that the assumption of a continuous viscoelastic spectrum for the composite material (section 3.2) was correct. There are some small differences towards the end of the relaxation, which illustrate the limits of the presented method. In fact, if viscoelastic elements with very low weights are included, oscillations can be observed in the homogenized spectra around the corresponding relaxation times. Therefore, the continuous function (Eq. 7) used to calculate the shear moduli of the Maxwell elements in section 3 was truncated for very long relaxation times, when the weights become small (less than $0.1 \%$ of the instantaneous elastic modulus). This leads to a relatively abrupt end of some components of the viscoelastic spectrum of the yarn, as can be seen from the transverse shear spectrum in Figure 2 (this phenomenon is less important for the matrix and for the axial shear spectrum of the yarn). The longest relaxation times are therefore not sufficiently well represented in the homogenized behavior. Nevertheless, these differences are small in the studied case, and the longest relaxation times become only relevant above $T_{g}$, which is not the most important temperature range for our applications (as above $T_{g}$, the stresses are almost immediately relaxed, this temperature range does not contribute much to the generation of residual stresses). The elastic homogenizations in the LC space needed to calculate the homogenized viscoelastic behavior were also carried out using a mesh with voxels of half 
the size as in the mesh shown in Figure 4 (i.e., 8 times as many voxels). The results are practically the same as with the mesh shown in Figure 5. We therefore conclude that this voxel mesh is fine enough for our purposes.

\subsection{Comparison with experimental observations}

Composites specimens were manufactured by resin injection molding (RTM) using the interlock woven reinforcement shown in Figure 4 and the epoxy resin tested by Courtois et al. [29], on which the model presented in section 2.2 was identified. Tensile tests were carried out according to the ASTM standard D3039 using a universal testing machine equipped with hydraulic grips and an oven. The specimen strain was measured using strain gauges, and the temperature controlled by thermocouples glued to the specimens. Acoustic emission sensors were placed on the specimens to detect the damage thresholds. The apparent elastic properties of the specimens were measured at different temperatures reaching from $-50^{\circ} \mathrm{C}$ to $200^{\circ} \mathrm{C}$, by determining the secant modulus of the stress-strain curve between strains of $1 \cdot 10^{-3}$ and $4 \cdot 10^{-3}$. It was ensured that the material is not damaged in the strain range, over which the elastic moduli are determined. Only fully cured specimens were tested. The experimental validation of the homogenization procedure is thus limited to the temperature dependence.

The tensile moduli were measured in direction parallel to the warp (index 1) and the weft (index 2) yarns, respectively. The in-plane shear moduli (indices 12) were measured by Iosipescu shear tests based on the ASTM standard D5379, while the out-of-plane shear moduli (indices 23 and 31) where measured using V-notched rail shear tests based on the ASTM standard D7078. The shear strain in the specimens was measured using strain gauge rosettes on both surfaces of the specimens. The apparent shear moduli were taken as the secant moduli between shear strains of $1 \cdot 10^{-3}$ and $4 \cdot 10^{-3}$. In each of the testing setups, the loading speed was adjusted such that a strain rate of $10^{-3} \mathrm{~s}^{-1}$ was measured with the strain gauges.

The homogenized viscoelastic behavior shown in Figure 5 was used to calculate the evolution of the apparent elastic properties of the composite for strain rates of $10^{-3} \mathrm{~s}$ and $10^{-4} \mathrm{~s}$. To this aim, the stress response of the homogenized model under a monotonically increasing uniaxial strain was calculated using the equations given in section 2.1. The apparent tensile moduli are determined from the resulting stress-strain curve in the same way as for the experimental data. Likewise, the apparent shear moduli are obtained by applying a monotonically increasing pure shear strain. The relaxation times of the homogenized viscoelastic model are calculated using Eq. 10 with the shift factors of the matrix (as described in Section 3.3), taking $T_{g}$ of the fully cured resin (see Table 1) as the reference temperature. In this way, the evolution of the apparent moduli with temperature can be calculated using the homogenized viscoelastic model of the composite.

The results are compared to the experimental observations in Figure 6. Due to confidentiality obligations, the numerical and experimental moduli are normalized by the tensile modulus in warp direction measured at $25^{\circ} \mathrm{C}$. The numerical results agree well with the experiments up to temperatures of about $120^{\circ} \mathrm{C}$, where the 
experimentally measured moduli start to decrease significantly due to the vicinity to the $T_{g}$ of the polymer matrix. On average the moduli are slightly underestimated up to $120^{\circ} \mathrm{C}$, which may be due to the use of periodic boundary conditions. However, other reasons are possible, like for example that the polymerization of the matrix may be influenced by the presence of the fibers (even if the same temperature cycle was applied to the composite and the resin specimens) or that the fiber volume fractions vary within the yarns (in our model, we assumed a constant fiber volume fraction everywhere in the warp yarns and a different constant fiber volume fraction everywhere in the weft yarns). Significant differences around the $T_{g}$ of the matrix are not surprising, since only very few experimental data was available to identify the model presented in section 2.2 at temperatures above $130^{\circ} \mathrm{C}$ (see [29]), and therefore, the matrix model is probably not very reliable in this temperature range. Above $T_{g}$, the matrix becomes very soft, such that interactions between the yarns become important. These interactions are not taken into account in the meso-scale composite model, which is based on a small strain formulation. Therefore, the calculated relaxed moduli are lower than observed experimentally. In fact, above $T_{g}$, the behavior of the composite is expected to become closer to that of the reinforcement without the matrix. On the whole, considering that we only used the properties of the constituents as input data (the viscoelastic model for the epoxy matrix identified independently, a voxelized FE mesh of the composite identified from $\mu$-CT images, and elastic properties for the carbon fibers that were proven to give accurate results at room temperature), the proposed method provides an excellent estimation of the evolution of the elastic properties of a composite with a complex $3 \mathrm{D}$ woven reinforcement with temperature.

\section{Conclusions}

A homogenization technique was proposed exploiting the linear relationship between stress and strain in a linear viscoelastic model in Schapery's integral form [45] after application of the LC transform. Under the assumption of continuous viscoelastic spectra of all constituents and the composite material, the same relaxation times were chosen for the constituents and the composite (one per decade covering the range of interest). The $\mathrm{LC}$ parameters $p$, at which the $\mathrm{LC}$ transform of the viscoelastic behavior of the constituents is calculated and the elastic homogenizations are carried out in the transformed space, were chosen at intermediate values with respect to the inverse of the relaxation times. This choice reduces the condition number of the equation system to be solved to calculate the homogenized viscoelastic behavior via the inverse of the LC transform and avoids oscillations in the homogenized spectra observed with other choices of $p$. In this case, the proposed homogenization technique consists of $N+3$ elastic homogenizations in the transformed space, where $N$ is the number of relaxation times of the constituents. Since in the matrix model, temperature $T$ and degree of cure $c$ only influence the relaxation times and the parameters $p$ are based on the inverse of the relaxation times, the LC transform matrix is independent of $T$ and $c$, and hence 
the same $T$ and $c$ dependence of the relaxation times is obtained for the homogenized behavior.

The proposed method was applied to the case of a composite made of a 3D interlock woven reinforcement and an epoxy matrix, whose viscoelastic behavior was determined experimentally elsewhere [29]. First, a homogenization at the microscopic scale was carried out to obtain the viscoelastic behavior of the warp and weft yarns. In a second step, these behaviors were used together with the matrix behavior and a mesoscopic RUC obtained from micro-CT images of the composite to calculate the homogenized viscoelastic behavior of the composite. Numerical comparison of the obtained homogenized behavior and full scale FE simulations show excellent agreement over the whole relaxation time scale, both at the microscopic and the mesoscopic scale.

The resulting homogenized viscoelastic model was used to predict the temperature dependence of the mechanical behavior of the composite between $-50^{\circ} \mathrm{C}$ and $200^{\circ} \mathrm{C}$. Good agreement with experimentally tested composite moduli was observed, in particular in the temperature range where the matrix behavior was properly identified. This result is very promising, as some major complexities, such as a varying cure cycle of the resin within the composite compared to pure resin specimens (due to the presence of the reinforcement) or fiber volume fractions that vary within different yarns were neglected.

As the model of the resin also includes the dependence of the viscoelastic behavior on degree of cure, it should be possible to estimate the viscoelastic properties of partially cured composites with the proposed homogenization method. To validate this aspect, composite specimens manufactured via interrupted cure cycles will be tested mechanically in a future study. The fully validated procedure can then be used to model of the generation of residual stresses during the curing process in composites with $3 \mathrm{D}$ textile reinforcements. Very promising results were already obtained by Benavente et al. [56] in using the presented homogenized model to predict the evolution of the distortion of composite plates and L-angles due to creep and relaxation phenomena linked to the viscoelastic nature of the matrix material.

\section{References}

[1] B. Fiedler, A. Hojo, S. Ochiai, The influence of thermal residual stresses on the transverse strength of CFRP using FEM, Composites Part A: Applied Science and Manufacturing 33 (10) (2002) 1323-1326.

[2] G. Maier, F. Hofmann, Performance enhancements of polymer-matrix composites by changing of residual stresses, Composites Science and Technology 68 (9) (2008) 2056-2065.

[3] E. Correa, F. París, V. Mantič, Effect of thermal residual stresses on the matrix failure under transverse compression at micromechanical level - A numerical and experimental study, Composites Part A: Applied Science and Manufacturing 43 (1) (2012) 87-94.

[4] S. Agius, M. Joosten, B. Trippit, C. Wang, T. Hilditch, Rapidly cured epoxy/anhydride composites: Effect of residual stress on laminate shear strength, Composites Part A: Applied Science and Manufacturing 90 (2016) 125-136.

[5] G. Fernlund, N. Rahman, R. Courdji, M. Bresslauer, A. Poursartip, K. Willden, K. Nelson, Experimental and numerical study of the effect of cure cycle, tool surface, geometry, and lay-up on the dimensional fidelity of autoclave-processed composite parts, Composites Part A: Applied Science and Manufacturing 33 (3) (2002) 341-351. 
[6] M. Wisnom, M. Gigliotti, N. Ersoy, M. Campbell, K. Potter, Mechanisms generating residual stresses and distortion during manufacture of polymer-matrix composite structures, Composites Part A: Applied Science and Manufacturing 37 (4) (2006) 522-529.

[7] N. Ersoy, K. Potter, M. Wisnom, M. Clegg, Development of spring-in angle during cure of a thermosetting composite, Composites Part A: Applied Science and Manufacturing 36 (12) (2005) 1700-1706.

[8] P. Causse, E. Ruiz, F. Trochu, Spring-in behavior of curved composites manufactured by Flexible Injection, Composites Part A: Applied Science and Manufacturing 43 (11) (2012) 1901-1913.

[9] E. Kappel, D. Stefaniak, D. Holzhüter, C. Hühne, M. Sinapius, Manufacturing distortions of a CFRP box-structure - A semi-numerical prediction approach, Composites Part A: Applied Science and Manufacturing 51 (2013) 89-98.

[10] D. A. Darrow, L. V. Smith, Isolating components of processing induced warpage in laminated composites, Journal of Composite Materials 36 (21) (2002) 2407-2419.

[11] C. Li, K. Potter, M. R. Wisnom, G. Stringer, In-situ measurement of chemical shrinkage of MY750 epoxy resin by a novel gravimetric method, Composites Science and Technology 64 (1) (2004) 55-64.

[12] C. Billotte, F. Bernard, E. Ruiz, Chemical shrinkage and thermomechanical characterization of an epoxy resin during cure by a novel in situ measurement method, European Polymer Journal 49 (11) (2013) 3548-3560.

[13] J. D. Russell, M. S. Madhukar, M. S. Genidy, A. Y. Lee, A new method to reduce cure-induced stresses in thermoset polymer composites, Part III: Correlating stress history to viscosity, degree of cure, and cure shrinkage, Journal of Composite Materials 34 (22) (2000) 1926-1947.

[14] Y. K. Kim, S. R. White, Stress relaxation behavior of 3501-6 epoxy resin during cure, Polymer Engineering \& Science 36 (23) (1996) 2852-2862.

[15] S. Simon, G. McKenna, O. Sindt, Modeling the evolution of the dynamic mechanical properties of a commercial epoxy during cure after gelation, Journal of Applied Polymer Science 76 (4) (2000) 495-508.

[16] D. O'Brien, P. Mather, S. White, Viscoelastic properties of an epoxy resin during cure, Journal of Composite Materials 35 (10) (2001) 883-904.

[17] E. Ruiz, F. Trochu, Numerical analysis of cure temperature and internal stresses in thin and thick RTM parts, Composites Part A: Applied Science and Manufacturing 36 (6) (2005) 806-826.

[18] N. Ersoy, T. Garstka, K. Potter, M. R. Wisnom, D. Porter, M. Clegg, G. Stringer, Development of the properties of a carbon fibre reinforced thermosetting composite through cure, Composites Part A: Applied Science and Manufacturing 41 (3) (2010) 401-409.

[19] T. Hobbiebrunken, B. Fiedler, M. Hop, S. Ochial, K. Schulte, Microscopic yielding of CF/epoxy composites and the effect on the formation of thermal residual stresses, Composites Science and Technology 65 (10) (2005) 1626-1635.

[20] A. Ding, S. Li, J. Sun, J. Wang, L. Zu, A thermo-viscoelastic model of process-induced residual stresses in composite structures with considering thermal dependence, Composite Structures 136 (2016) 34-43.

[21] M. Benavente, L. Marcin, A. Courtois, M. Lévesque, E. Ruiz, Viscoelastic distortion in asymmetric plates during post curing, Composites Part A: Applied Science and Manufacturing 103 (2017) 122-130.

[22] J. W. Kim, J. H. Lee, H. G. Kim, H. S. Kim, D. G. Lee, Reduction of residual stresses in thick-walled composite cylinders by smart cure cycle with cooling and reheating, Composite Structures 75 (1-4) (2006) 261-266.

[23] M. Biot, Theory of stress-strain relations in anisotropic viscoelasticity and relaxation phenomena, Journal of Applied Physics 25 (11) (1954) 1385-1391.

[24] P. Sunderland, W. Yu, J.-A. Månson, A thermoviscoelastic analysis of process-induced internal stresses in thermoplastic matrix composites, Polymer Composites 22 (5) (2001) 579-592.

[25] J. Svanberg, J. Holmberg, Prediction of shape distortions Part I. FE-implementation of a path dependent constitutive model, Composites Part A: Applied Science and Manufacturing 35 (6) (2004) 711-721. 
[26] S. Clifford, N. Jansson, W. Yu, V. Michaud, J.-A. Manson, Thermoviscoelastic anisotropic analysis of process induced residual stresses and dimensional stability in real polymer matrix composite components, Composites Part A: Applied Science and Manufacturing 37 (4) (2006) 538-545545.

[27] J. Zhang, M. Zhang, S. Li, M. Pavier, D. Smith, Residual stresses created during curing of a polymer matrix composite using a viscoelastic model, Composites Science and Technology 130 (2016) 20-27.

[28] M. Zarrelli, A. Skordos, I. Partridge, Toward a constitutive model for cure-dependent modulus of a high temperature epoxy during the cure, European Polymer Journal 46 (8) (2010) 1705-1712.

[29] A. Courtois, M. Hirsekorn, M. Benavente, A. Jaillon, L. Marcin, E. Ruiz, M. Lévesque, Viscoelastic behavior of an epoxy resin during cure below the glass transition temperature: Characterization and modeling, Journal of Composite Materials (2018), in press.

[30] A. Mouritz, M. Bannister, P. Falzon, K. Leong, Review of applications for advanced three-dimensional fibre textile composites, Composites Part A: Applied Science and Manufacturing 30 (12) (1999) 1445-1461.

[31] R. Kamiya, B. A. Cheeseman, P. Popper, T.-W. Chou, Some recent advances in the fabrication and design of threedimensional textile preforms: a review, Composites Science and Technology 60 (1) (2000) 33-47.

[32] M. Ansar, W. Xinwei, Z. Chouwei, Modeling strategies of 3D woven composites: A review, Composite Structures 93 (8) (2011) 1947-1963.

[33] A. Mountasir, G. Hoffmann, C. Cherif, M. Löser, K. Großmann, Competitive manufacturing of 3D thermoplastic composite panels based on multi-layered woven structures for lightweight engineering, Composite Structures 133 (2015) 415-424.

[34] R. Gerlach, C. R. Siviour, J. Wiegand, N. Petrinic, In-plane and through-thickness properties, failure modes, damage and delamination in $3 \mathrm{D}$ woven carbon fibre composites subjected to impact loading, Composites Science and Technology 72 (3) (2012) 397-411.

[35] P. Turner, T. Liu, X. Zeng, Collapse of 3D orthogonal woven carbon fibre composites under in-plane tension/compression and out-of-plane bending, Composite Structures 142 (2016) 286-297.

[36] S. V. Lomov, D. S. Ivanov, I. Verpoest, M. Zako, T. Kurashiki, H. Nakai, S. Hirosawa, Meso-FE modelling of textile composites: Road map, data flow and algorithms, Composites Science and Technology 67 (9) (2007) 1870-1891.

[37] R. Brenner, R. Masson, O. Castelnau, A. Zaoui, A "quasi-elastic" affine formulation for the homogenised behaviour of nonlinear viscoelastic polycrystals and composites, European Journal of Mechanics - A/Solids 21 (6) (2002) 943-960.

[38] M. Lévesque, M. D. Gilchrist, N. Bouleau, K. Derrien, D. Baptiste, Numerical inversion of the Laplace-Carson transform applied to homogenization of randomly reinforced linear viscoelastic media, Computational Mechanics 40 (4) (2007) 771789 .

[39] J. M. Ricaud, R. Masson, Effective properties of linear viscoelastic heterogeneous media: Internal variables formulation and extension to ageing behaviours, International Journal of Solids and Structures 46 (7-8) (2009) 1599-1606.

[40] C. Pichler, R. Lackner, Upscaling of viscoelastic properties of highly-filled composites: Investigation of matrix-inclusiontype morphologies with power-law viscoelastic material response, Composites Science and Technology 69 (14) (2009) 2410-2420.

[41] A. El Mourid, R. Ganesan, M. Lévesque, Comparison between analytical and numerical predictions for the linearly viscoelastic behavior of textile composites, Mechanics of Materials 58 (2013) 69-83.

[42] S. Beurthey, A. Zaoui, Structural morphology and relaxation spectra of viscoelastic heterogeneous materials, European Journal of Mechanics - A/Solids 19 (1) (2000) 1-16.

[43] A. Thionnet, M. Salvia, C. Turki, J. Renard, Prévision du comportement en fluage viscoélastique endommageable des structures à matrice organique, Revue des Composites et des Matériaux Avancés 14 (3) (2004) 331-351.

[44] S. Sawant, A. Muliana, A thermo-mechanical viscoelastic analysis of orthotropic materials, Composite Structures 83 (1) (2008) 61-72. 
[45] R. Schapery, On the characterization of nonlinear viscoelastic materials, Polymer Engineering \& Science 9 (4) (1969) $295-310$.

[46] N. Tschoegl, W. G. Knauss, I. Emri, Poisson's ratio in linear viscoelasticity - A critical review, Mechanics of TimeDependent Materials 6 (1) (2002) 3-51.

[47] D. O'Brien, N. Sottos, S. White, Cure-dependent viscoelastic poisson's ratio of epoxy, Experimental Mechanics 47 (2) (2007) 237-249.

[48] M. Sadeghinia, K. Jansen, L. Ernst, Characterization and modeling the thermo-mechanical cure-dependent properties of epoxy molding compound, International Journal of Adhesion and Adhesives 32 (2012) 82-88.

[49] J. Pascault, J. Williams, Relationships between glass transition temperature and conversion, Polymer Bulletin 24 (1990) $115-121$.

[50] G. Grail, M. Hirsekorn, A. Wendling, G. Hivet, R. Hambli, Consistent Finite Element mesh generation for meso-scale modeling of textile composites with preformed and compacted reinforcements, Composites Part A: Applied Science and Manufacturing 55 (2013) 143-151.

[51] A. Doitrand, C. Fagiano, F. Irisarri, M. Hirsekorn, Comparison between voxel and consistent meso-scale models of woven composites, Composites Part A: Applied Science and Manufacturing 73 (2015) 143-154.

[52] N. Isart, B. El Said, D. Ivanov, S. Hallett, J. Mayugo, N. Blanco, Internal geometric modelling of 3D woven composites: A comparison between different approaches, Composite Structures 132 (2015) 1219-1230.

[53] J. Schneider, G. Hello, Z. Aboura, M. L. Benzeggagh, D. Marsal, A meso-FE voxel model of an interlock woven composite, in: Proceedings of the 17th International Conference on Composite Materials (ICCM17), Edinburgh, Scotland, 2009.

[54] G. Hello, J. Schneider, Z. Aboura, Numerical simulations of woven composite materials with voxel-FE models, in: Proceedings of the 16th European Conference on Composite Materials (ECCM16), Seville, Spain, 2014.

[55] N. Naouar, E. Vidal-Salle, J. Schneider, E. Maire, P. Boisse, 3D composite reinforcement meso F.E. analyses based on X-ray computed tomography, Composite Structures 132 (2015) 1094-1104.

[56] M. Benavente, L. Marcin, A. Courtois, M. Lévesque, E. Ruiz, Numerical analysis of viscoelastic process-induced residual distortions during manufacturing and post-curing, Composites Part A: Applied Science and Manufacturing 107 (2018) $205-216$.

\section{Acknowledgements}

The research presented in this article was funded by the Safran Group, France. 


\begin{tabular}{ccccc}
\hline$A[\mathrm{MPa}]$ & $B[\mathrm{MPa}]$ & $\log _{10} \tau_{\text {peak }}$ & $l_{\text {peak }}$ & $G_{\infty}[\mathrm{MPa}]$ \\
302.6 & 50.85 & -0.3380 & 1.3638 & 2.089 \\
\hline$\nu_{0}$ & $H(c)[\mathrm{kJ} / \mathrm{mol}]$ & $\lambda$ & $T_{g 0}\left[{ }^{\circ} \mathrm{C}\right]$ & $T_{g 1}\left[{ }^{\circ} \mathrm{C}\right]$ \\
0.35 & $288.9-111.4 c$ & 0.2603 & 155.0 & 46.78 \\
\hline
\end{tabular}

Table 1: Parameters of the viscoelastic model for the epoxy resin.

\begin{tabular}{ccccc}
\hline$E_{L}[\mathrm{GPa}]$ & $E_{T}[\mathrm{GPa}]$ & $G_{L T}[\mathrm{GPa}]$ & $\nu_{L T}$ & $\nu_{T T}$ \\
276.0 & 10.3 & 27.9 & 0.26 & 0.3 \\
\hline
\end{tabular}

Table 2: Transverse isotropic elastic properties of the fibers: $E$ are Young's moduli, $G$ the shear modulus, and $\nu$ the Poisson ratios. The index $L$ indicates the direction parallel to the fiber, $T$ indicates the transverse direction. 


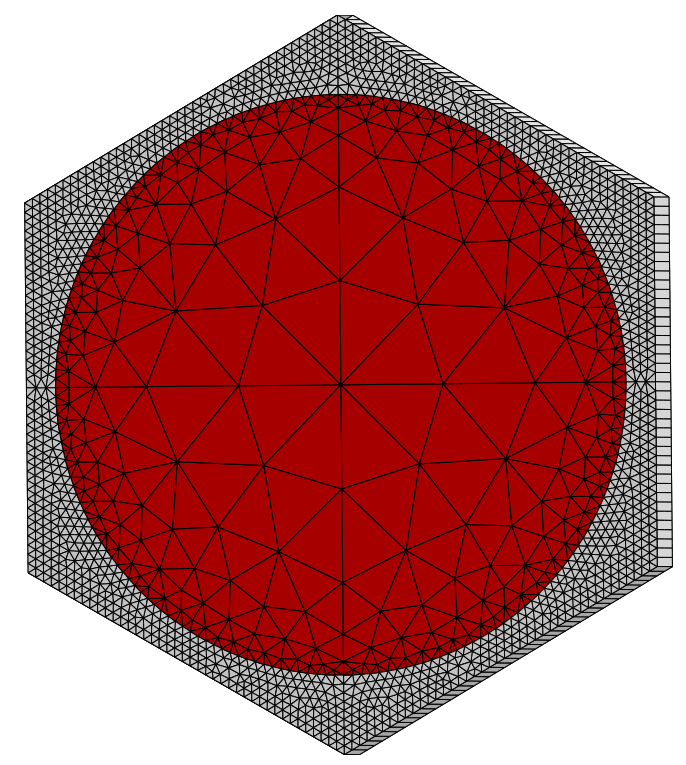

Figure 1: FE mesh of the micro-scale RUC (fiber in red, matrix in gray).

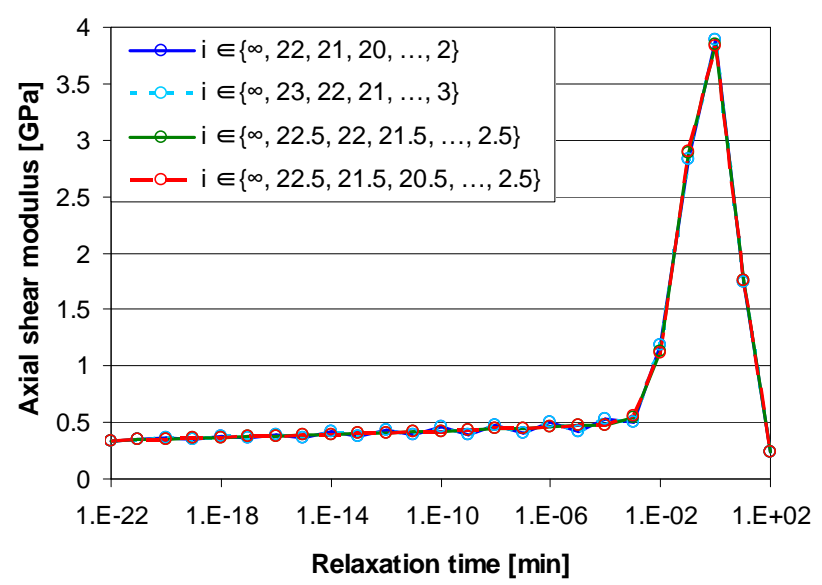

(a)

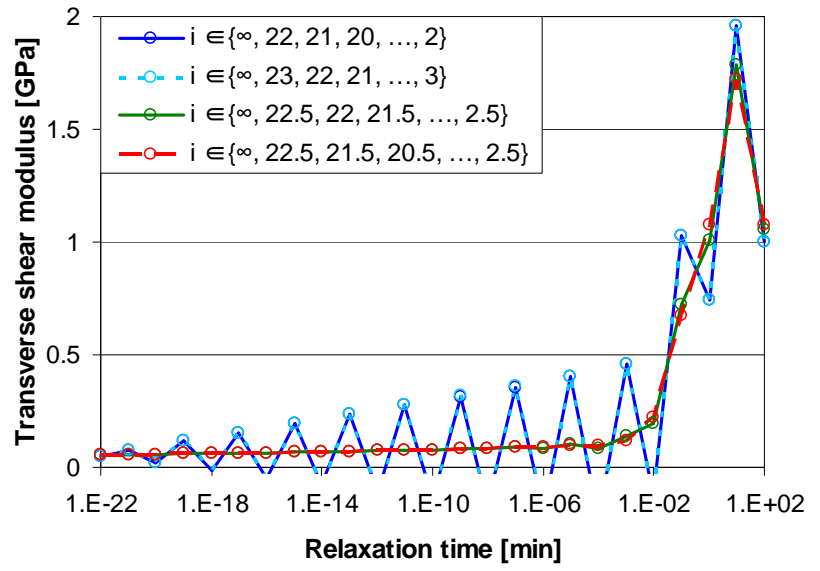

(b)

Figure 2: (a) homogenized axial shear moduli $\left(G_{L T}(\tau)\right)$ and (b) homogenized transverse shear moduli $\left(G_{T T}(\tau)\right)$ of the Maxwell elements as a function of the relaxation time $\tau$ for the homogenized viscoelastic behavior of the warp yarns for different choices of $p_{i}=10^{i} \mathrm{~min}^{-1}$. The corresponding condition numbers of the LC transform matrix $\mathcal{L}$ are (in order of appearance): 1240.9, 1286.1, 299.3, and 221.0. 


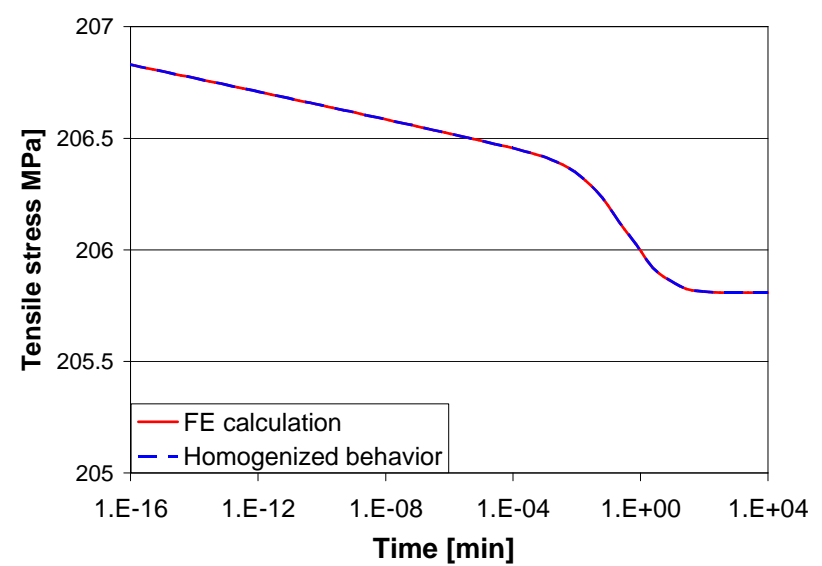

(a)

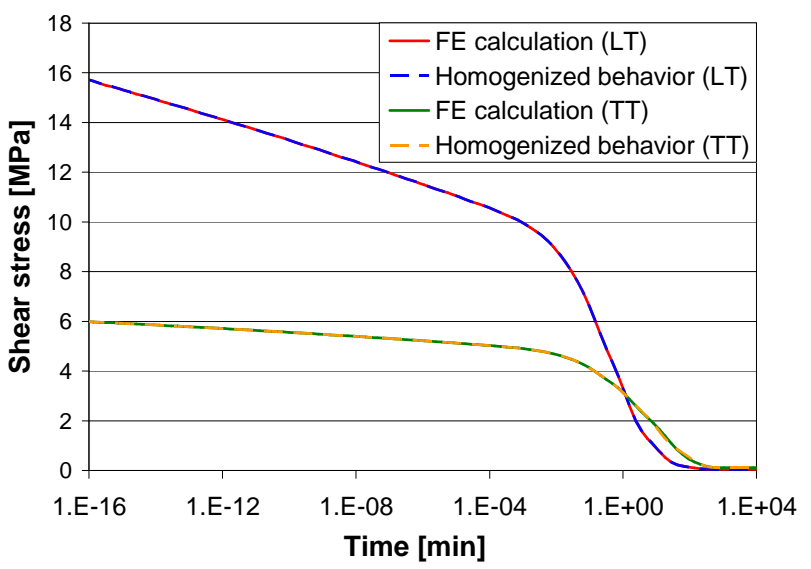

(c)

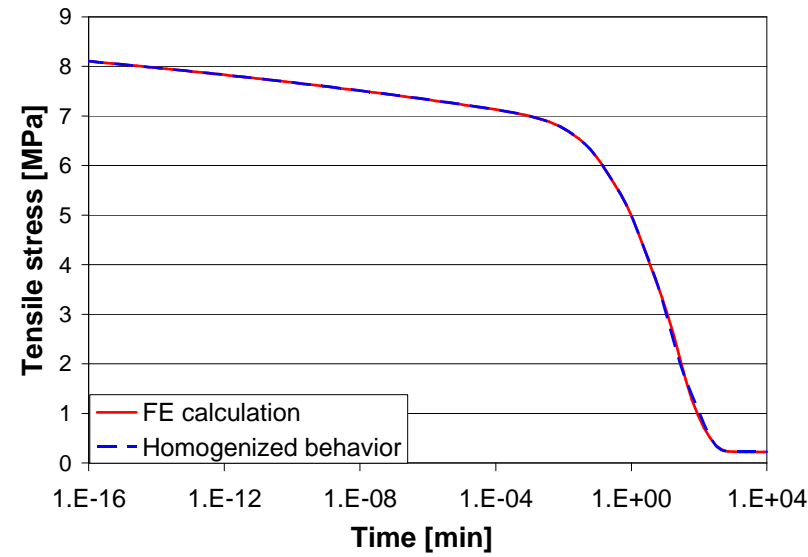

(b)

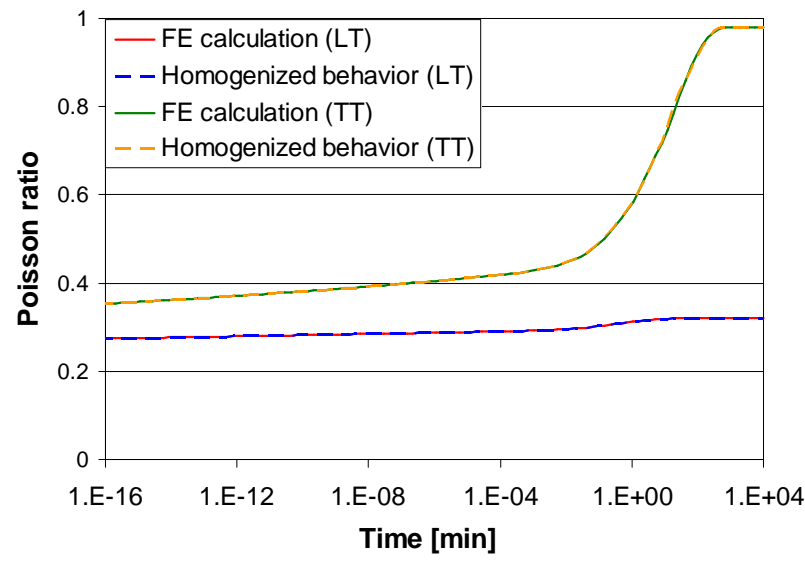

(d)

Figure 3: Comparison between the stress response of the homogenized viscoelastic model for the warp yarns and the FE simulation using the RUC shown in Figure 1: stress in loading direction under constant tensile strain in axial (a) and transverse (b) direction with respect to the fiber, (c) shear stress evolution under constant shear strain in the axial (LT) and the transverse (TT) plane, and (d) evolution of the ratio between the negative transverse strain and the constant applied tensile strain in time axial (LT) and transverse (TT) direction. 


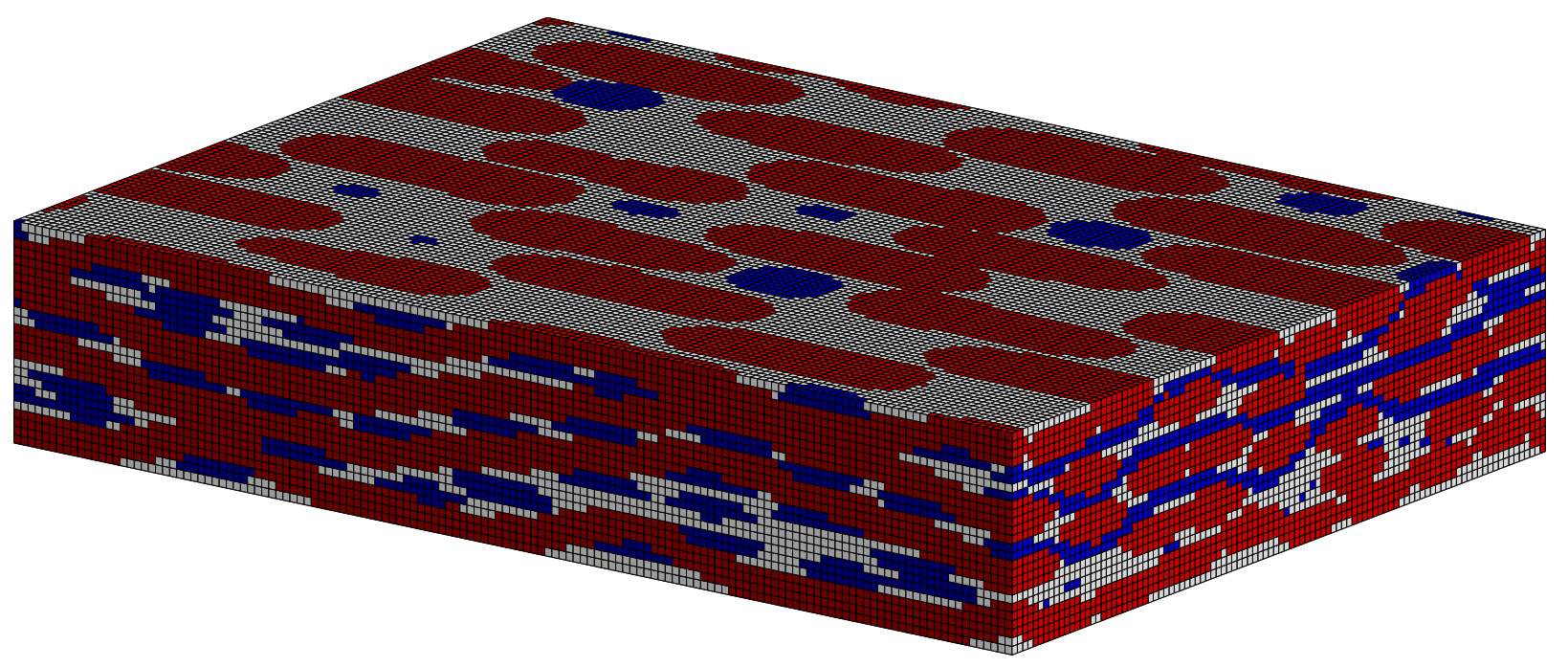

Figure 4: Voxel mesh of the meso-scale RUC of the composite (warp yarns in red, weft yarns in blue, matrix in gray). 


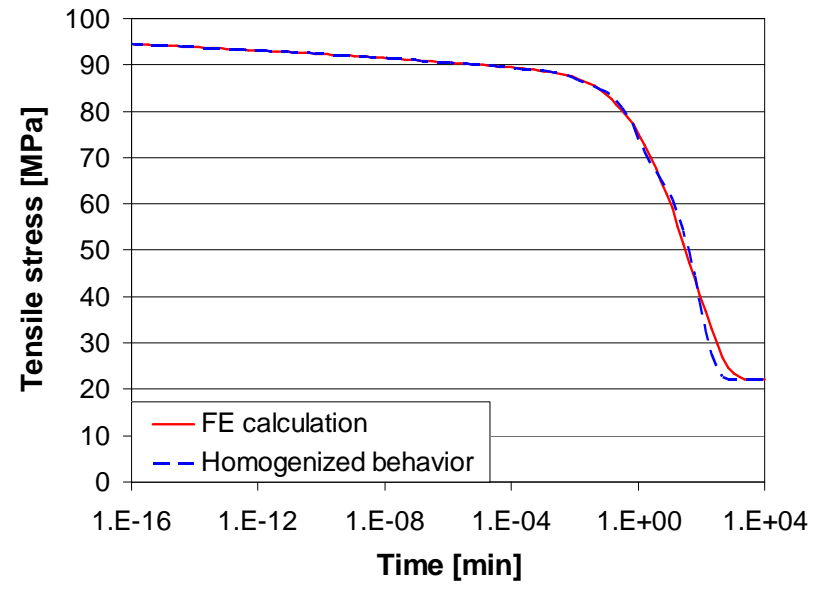

(a)

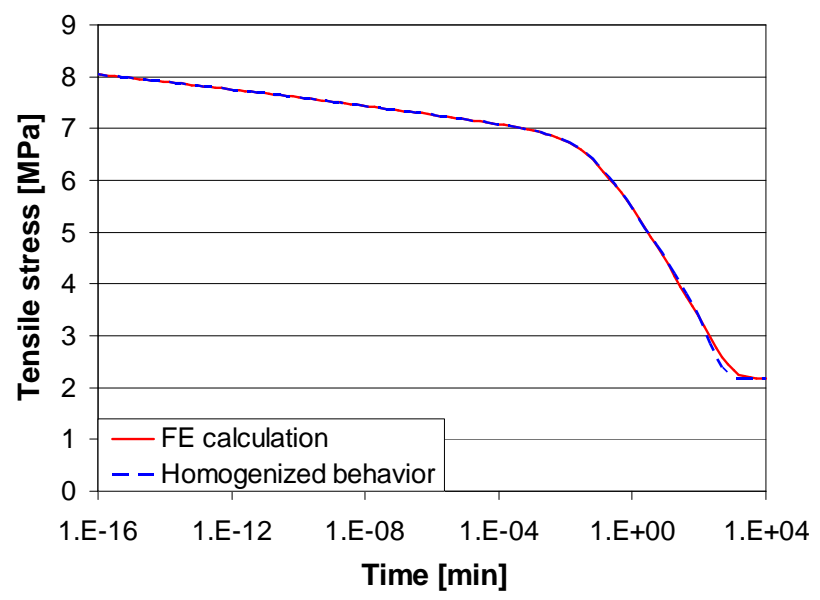

(c)

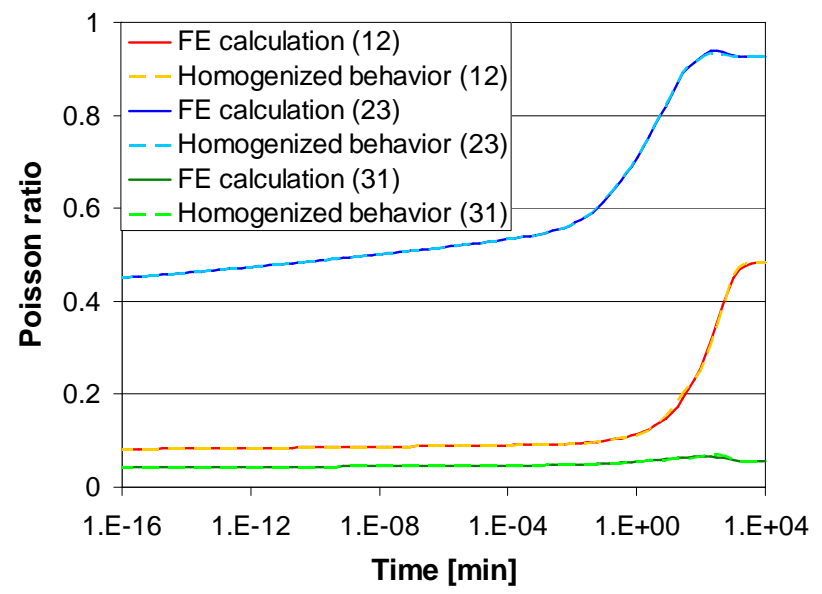

(e)

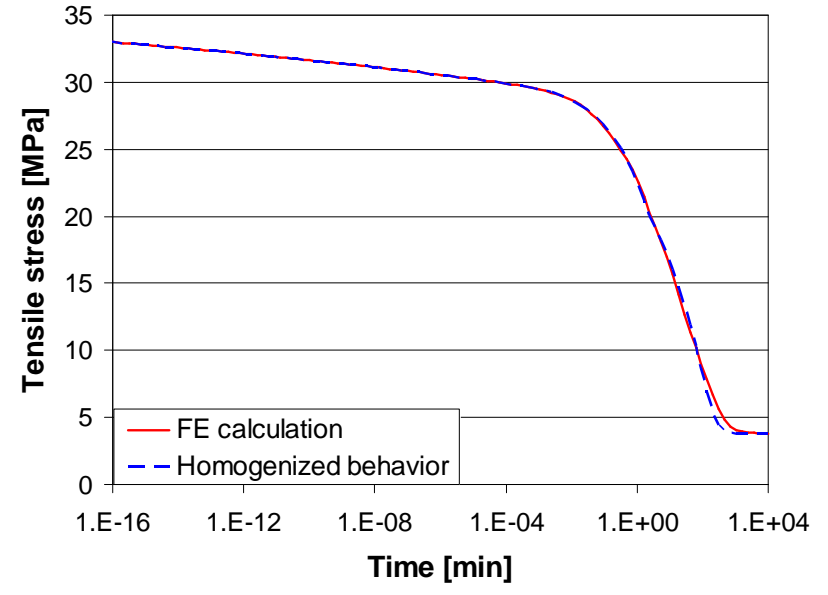

(b)

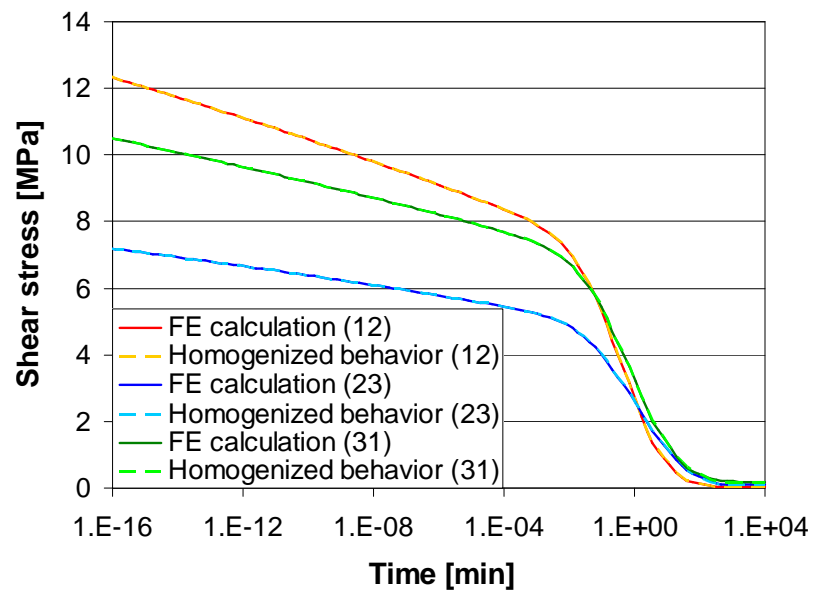

(d)

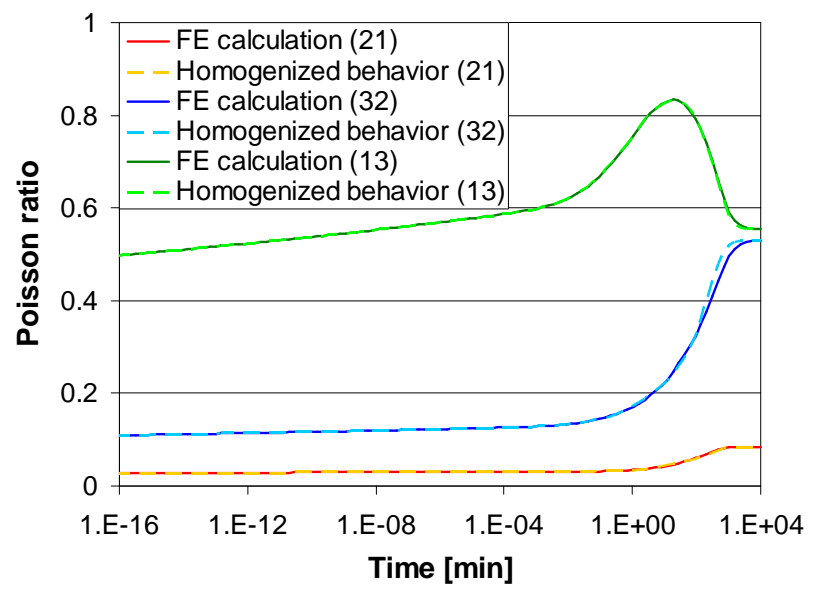

(f)

Figure 5: Comparison between the stress response of the homogenized viscoelastic model for the composite and the FE simulation using the RUC shown in Figure 4: stress in loading direction under constant tensile strain in (a) warp, (b) weft), and (c) out-of-plane direction, (d) shear stress evolution under constant shear strain, and (e) and (f) evolution of the Poisson ratios under constant tensile strain. 


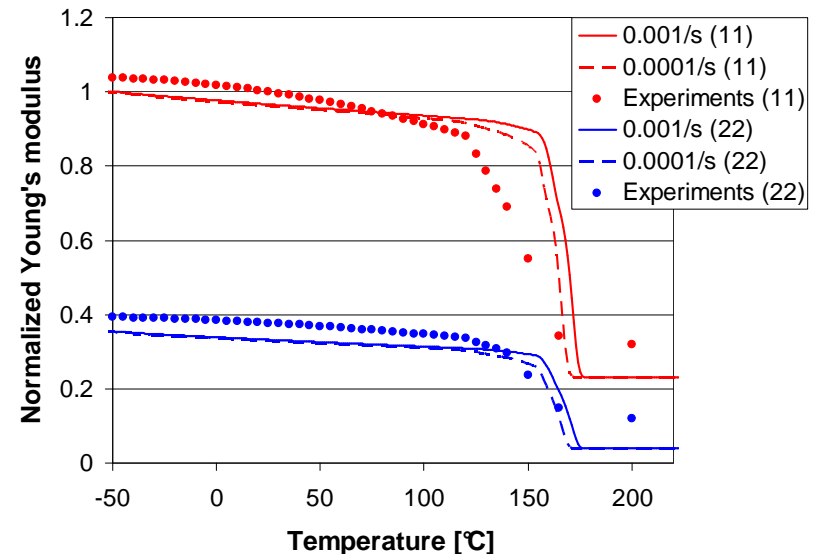

(a)

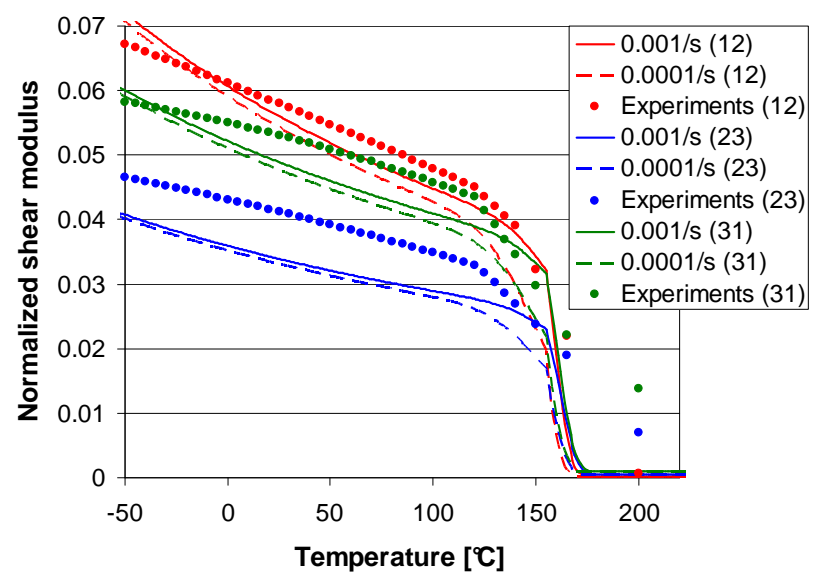

(b)

Figure 6: Secant moduli calculated using the homogenized viscoelastic behavior of the composite at a strain rate of $0.001 \mathrm{~s}^{-1}$ (continuous lines) and $0.0001 \mathrm{~s}^{-1}$ (dashed lines) compared with experimentally measured moduli of the composite at a strain rate of $0.001 \mathrm{~s}^{-1}$ (dots). The moduli are normalized by the experimentally measured tensile modulus in warp direction at $25^{\circ} \mathrm{C}$. 